\title{
Analyse de réseaux sur les personnages des Confessions de Jean-Jacques Rousseau
}

YANNICK ROCHAT

FRÉDÉRIC KAPLAN

Dans cet article, nous présentons les méthodes de l'analyse de réseaux littéraires. Cette discipline se situe à la croisée de la narratologie, du « distant reading » et de l'analyse des réseaux sociaux. Nous l'illustrons par une analyse sur Les Confessions de Jean-Jacques Rousseau utilisant le concept de centralité, et permettant ainsi de caractériser certains aspects des rôles des personnages du récit sur la base de leurs co-occurrences dans le texte.

\section{Introduction}

Dans une oeuvre littéraire, les « unités constitutives » de l'histoire sont les événements, personnages, lieux et les temps de l'action (Bal, 1984). La compréhension des structures formées par les apparitions et interactions des personnages en particulier est souvent secondaire dans l'analyse, ou partiellement explorée lorsqu'une étude porte sur la composition d'une intrigue et l'intrication des personnages dans celle-ci.

Dans cet article, nous proposons une analyse de réseaux des personnages du texte sur la base de leurs co-occurrences. Les personnages sont saisis indépendamment de l'intrigue, mais en fonction de leurs apparitions dans l'ouvrage. L'index des personnages nous fournit cette information. Sur la base de celuici, nous générons un réseau représentant le système des personnages (Woloch, 2003).

Nous appliquons les méthodes de l'analyse de réseaux littéraires ${ }^{1}$ (Moretti, 2011; Elson, 2012; Sack, 2013) aux Confessions de Rousseau. Les Confesions est un texte de fiction autobiographique (Lejeune, 1975) composé d'un grand nombre de personnages. Nous basons nos travaux sur l'édition des œuvres complètes parue chez Slatkine en 2012. L'index de cette édition identifie 584 personnages sur un total de 774 pages. Rousseau n'apparaît pas lui-même dans l'index.

Nous faisons usage du concept de centralité, utilisé dans le cadre de l'analyse des réseaux sociaux. La centralité correspond à une famille de mesures mettant en évidence l'une ou l'autre propriété structurelle des sommets du réseau. Les indices de centralité permettent de définir des types de comportements ou de détecter des rôles joués par les noeuds du réseau, c'est-à-dire, dans notre contexte, les personnages tels qu'ils apparaissent dans l'oeuvre. La prise en compte simultanée de plusieurs indices de centralité permet de proposer une caractérisation des personnages.

Dans les parties qui suivent, nous commençons par définir l'analyse de réseaux littéraires. L'objectif est d'étudier l'organisation des personnages dans le texte, comment leurs rôles au niveau de l'histoire et du récit se trouvent trans-

\footnotetext{
${ }^{1}$ L'analyse de réseaux littéraires, ou « literary network analysis », a un sens très large. Elle inclut par exemple l'étude par la mise en réseaux des influences dans l'histoire de la littérature (Jockers, 2013).
} 
formés, en pratique, en passant au niveau de la narration. Puis, nous présentons la méthode de construction du réseau des co-occurrences. Nous justifions nos choix et décrivons les étapes nécessaires pour l'obtenir, et commentons ensuite les propriétés de ce réseau. Finalement, nous calculons les indices de centralité des personnages et montrons comment, en composant ces indices, l'utilisation de ce concept permet de caractériser les rôles narratifs des personnages d'un roman.

\section{Analyse de réseaux littéraires}

\section{$2.1 \quad$ Définition}

L'analyse de réseaux littéraires étudie l'organisation des entités narratives au moyen de la théorie des graphes (Berge, 1958). Un réseau est constitué de sommets représentant les entités narratives et d'arêtes qui les lient entre elles le cas échéant. La présence ou l'absence d'une arête entre deux sommets dépend de la méthode d'identification des relations. Les méthodes d'analyse sont inspirées de celles de l'analyse des réseaux sociaux (Wasserman et Faust, 1994; Borgatti et al., 2009).

Dans le reste de ce travail, les entités narratives que nous étudions sont les personnages.

\section{$2.2 \quad$ Exemples}

Il existe de nombreux exemples d'extractions et d'études de réseaux de personnages tirés d'oeuvres littéraires. Les domaines dans lesquels ces recherches sont conduites comprennent les lettres et la théologie, tout comme la physique statistique et l'informatique. Nous remarquons une augmentation de la popularité de ce type de recherche.

Parmi les travaux existants, (Pentland et Feldman, 2007; Gil et al., 2011; Elson, 2012; Corman et al., 2013; Bolioli et al., 2013; Nalisnick et Baird, 2013a,b) s'intéressent à l'étape préliminaire de construction du réseau, notamment grâce à des méthodes d'extraction automatique des relations entre personnages, voire des contextes de l'action ou des relations. D'autres se focalisent sur l'analyse du ou des réseaux obtenus, et éventuellement des rôles des personnages (Stiller et al., 2003; Moretti, 2011; Carron et Kenna, 2012; Agarwal et al., 2012; Sack, 2012, 2013; Grandjean, 2013; Sparavigna, 2013). À plus grande échelle, (Alberich et al., 2002; Gleiser, 2007; Rydberg-Cox, 2011; Hutchinson et al., 2012) proposent des analyses de réseaux de personnages à partir d'ensembles de récits tirés chacun d'un univers cohérent, par exemple des sagas islandaises (Carron et Kenna, 2013).

En physique statistique, l'utilisation de tels réseaux sert d'abord à l'expérimentation de méthodes algorithmiques (Newman et Girvan, 2004). Le réseau n'est plus un objet narratif. Donald E. Knuth l'illustre à propos d'un célèbre jeu de données tiré des Misérables de Victor Hugo (Knuth, 2013) :

Since the data files were prepared by hand, they are subject to hu- 
man error. [...] They are intended simply as forever-frozen examples of typical data that is more or less accurate.

In particular, I recently learned that I forgot to include any connection between Fantine and her infant daughter Cosette, when I summarized the encounters between the characters of Les Misérables in the data file jean.dat. Fantine and Cosette appear together in chapter 1.4.1 (and then, of course - alas - they never see each other again). [...] However, I shall never update the file jean.dat, because it is "correct by definition."

\subsection{Le système des personnages}

Nous montrons que la construction d'un réseau de personnages à partir d'une oeuvre littéraire s'inscrit dans le cadre de la théorie des personnages définie par Alex Woloch (Woloch, 2003). Dans ce travail, nous fournissons à cette théorie un outil - l'analyse des réseaux littéraires - permettant de conduire une telle analyse de manière quantitative.

Tout d'abord, nous définissons l'espace du personnage :

That particular and charged encounter between an individual human personality and a determined space and position within the narrative as a whole. (Woloch, 2003, p.14)

L'espace du personnage est la projection d'un individu de l'histoire ${ }^{2}$ dans le récit ${ }^{3}$. Par exemple, on attend de l'espace du personnage protagoniste qu'il soit plus riche que l'espace d'un personnage secondaire. La narration implique que les scènes où apparaît un personnage secondaire soient plus rares, donc qu'une partie de l'information existante dans l'histoire soit omise ou réduite dans le récit. Alex Woloch forme ensuite l'ensemble de tous les espaces de personnages. Il le nomme le système des personnages et le définit comme :

The arrangement of multiple and differentiated character-spaces differentiated configurations and manipulations of the human figure - into a unified narrative structure. (Woloch, 2003, p. 14)

Dans le même temps, il définit une opération sur le système des personnages qui s'apparente à une forme mixte des concepts de réunion et d'intersection tels qu'on les comprend dans le domaine de la théorie des ensembles :

For the character-system offers not simply many interacting individuals but many intersecting character-spaces, each of which encompasses an embedded interaction between discretely implied person and the dynamicaly elaborated narrative form. (Woloch, 2003, p.17)

Cette approche topologique ${ }^{4}$ fournit un cadre théorique bien défini pour comprendre de quelle manière la narration transforme l'individu du monde virtuel

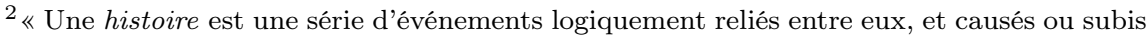
par des acteurs. »(Bal, 1984, p.4)

3 «Un récit est le signifié d'un texte narratif.» (Bal, 1984, p.4) « L'histoire ordonnée, structurée est le récit. » (Bal, 1984, p.8)

${ }^{4}$ Topologique car nous avons défini un ensemble accompagné d'un système de partitions de
} 
qu'est l'histoire en un personnage du récit. Nous étudions une fonction particulière de la narration, qui est son action sur l'individu, l'acteur, le personnage. À ce stade, pour décoder le rôle narratif du personnage, il est nécessaire de considérer sa position dans le flot du récit relativement aux entités narratives, dont, en particulier, les autres personnages :

[...] all character-spaces inevitably point us toward the charactersystem, since the emplacement of a character within the narrative form is largely comprised by his or her relative position vis-à-vis other characters. (Woloch, 2003, p.18)

Nous représentons le système des personnages à l'aide de l'analyse des réseaux littéraires. Celle-ci étudie la structure formée par les personnages, leurs relations et l'organisation de ces relations. Nous pouvons ensuite déduire une partie des propriétés narratives d'un personnage à partir de sa position dans le réseau des interactions des personnages.

\subsection{Centralité}

La centralité quantifie des propriétés structurelles des sommets du réseau interprétées comme des formes d'importance au sein de celui-ci (Hennig et al., 2012). Par exemple, la centralité de degré mesure le nombre de connexions d'un sommet, et donc l'intrication de l'espace d'un personnage dans les espaces d'autres personnages. La centralité d'intermédiarité, quand à elle, mesure la fréquence de placement d'un personnage sur les cheminements narratifs qui permettent de passer d'un personnage à un autre à travers le réseau des cooccurrences. Ainsi, entre autres possibilités, elle permet de mettre en évidence les personnages dont le rôle est central, ou ceux dont le rôle est de faire avancer le récit.

L'historique du développement du concept de centralité est de moindre importance ici en comparaison des questions soulevées depuis ses origines, à savoir, entre autres, "que mesure la centralité?", ou "comment utiliser et comparer ces mesures ?" (Sabidussi, 1966; Ruhnau, 2000; Koschützki et al., 2005; Borgatti, 2005; Borgatti et Everett, 2006; Brandes et al., 2012). De nombreuses formules mathématiques, valables sur les réseaux, peuvent être considérées comme des indices de centralité. En l'absence d'une théorie de la centralité qui uniformise ces formules et permettrait de travailler dans un cadre mathématique clairement défini, c'est un article de Linton C. Freeman présentant les centralités de degré, de proximité et d'intermédiarité (Freeman, 1978) et les travaux de Phillip Bonacich présentant la centralité par vecteurs propres (Bonacich, 1972, 1987) qui font référence et permettent de considérer ces quatre indices comme canoniques.

\subsubsection{Degré}

La centralité de degré d'un sommet s'exprime généralement par la somme des arêtes incidentes, ou de manière équivalente par la somme des sommets adja-

cet ensemble lui-même muni de règles d'union et d'intersection. S'il s'agissait d'un véritable espace topologique, l'union tout comme l'intersection d'espaces de personnages formeraient des espaces de personnages à leurs tours. Cette question n'est pas traitée dans le cadre dans ce travail. 
cents. C'est un indice ne prenant en compte que les éléments les plus proches du sommet, et ignorant le reste de la structure du réseau. Exprimé dans les termes de (Degenne et Forsé, 1994) :

La centralité de degré privilégie le point de vue local et mesure l'activité ou la capacité de communication ou d'échange de chaque individu au sein du réseau, en ne tenant pas compte de sa capacité à contrôler ces communications.

C'est un indice simple à mesurer et dont la lecture est intuitive. Mathématiquement, pour un sommet $x_{i}$ dans un réseau d'ordre ${ }^{5} n$ non-dirigé dont la matrice d'adjacence est $\left\{a_{i j}\right\}_{1 \leq i, j \leq n}$, la centralité de degré est :

$$
C_{D}\left(x_{i}\right)=\sum_{j} a_{i j}
$$

\subsubsection{Proximité}

La centralité de proximité consiste à évaluer la distance séparant un sommet de tous les autres. Cet indice est à l'origine du concept même de centralité (Bavelas, 1950). La centralité de proximité mesure l'effort nécessaire à un acteur du réseau pour atteindre tous les autres acteurs, par exemple lors de la diffusion d'une information. Dans l'analyse de réseaux littéraires appliquée au personnage, cet indice propose une forme de proximité narrative d'un personnage avec tous les autres. Pour ce travail, nous utilisons la centralité harmonique, dont les propriétés sont très proches et les résultats fortement corrélés avec la centralité de proximité (Rochat, 2009; Boldi et Vigna, 2013), mais dont la variance plus grande offre une meilleure lisibilité. Nous la définissons, pour un sommet $x_{i}$, par

$$
c_{H}\left(x_{i}\right)=\sum_{i \neq j} \frac{1}{\operatorname{dist}\left(x_{i}, x_{j}\right)}
$$

avec $\operatorname{dist}\left(x_{i}, x_{j}\right)$ la distance du sommet $x_{i}$ au sommet $x_{j}$.

\subsubsection{Intermédiarité}

La centralité d'intermédiarité mesure la propension d'un nœud à apparaître sur les plus courts chemins reliant les sommets du réseau. En sciences sociales tout comme dans des applications aux réseaux informatiques, les valeurs obtenues mettent en évidence les acteurs ou objets qui contrôlent l'information, ou par lesquels celle-ci transite.

Dans un réseau littéraire, la centralité d'intermédiarité met en évidence les personnages apparaissant dans des événements du récit communs à plusieurs personnages. S'il n'y a qu'un seul personnage dans ce cas, sa mesure de centralité d'intermédiarité sera significativement plus élevée que celles de ses voisins. Elle est donnée par

$$
c_{B}\left(x_{i}\right)=\sum \sum \frac{g_{j k}(i)}{g_{j k}}
$$

avec $g_{j k}$ le nombre de plus courts chemins allant du sommet $x_{j}$ au sommet $x_{k}$, et $g_{j k}(i)$ le nombre de plus courts chemins allant du sommet $x_{j}$ au sommet $x_{k}$

\footnotetext{
${ }^{5}$ En théorie des graphes, l'ordre est donné par le nombre de sommets.
} 
et contenant le sommet $x_{i}$. La double somme est calculée sur toutes les paires $(j, k)$ telles que $j \neq i \neq k$ et $j<k$.

\subsubsection{Par vecteurs propres}

La centralité par vecteurs propres ${ }^{6}$ attribue aux sommets une valeur tenant compte des centralités des sommets adjacents du réseau. Ainsi, il n'est pas possible de calculer la valeur de l'indice pour un sommet sans connaître celles des autres, et vice versa. Ce problème est résolu par l'utilisation de méthodes de l'algèbre linéaire ${ }^{7}$.

Cet indice mesure l'importance d'un sommet en fonction de celle de ses voisins et propose, à l'instar des centralités de proximité et d'intermédiarité, une description des sommets basée sur l'ensemble de la structure du réseau. Il est sensible à l'agglomération des sommets entre eux. Dans le contexte d'une étude structurelle du personnage, ceux qui ont le plus de connexions, tout en étant reliés aux personnages les plus importants, obtiennent de plus hautes valeurs. Les mesures obtenues ne permettent pas de délimiter des groupes de personnages fortement connectés ${ }^{8}$, mais mettent en évidence les personnages qui sont aux centres de ces groupes.

\section{Le réseau des co-occurrences}

Dans cette section, nous décrivons le processus de génération du réseau des cooccurrences des personnages. Les choix effectués sont à prendre en compte lors de l'interprétation des résultats de l'analyse des centralités. Par exemple, les mesures de l'importance des personnages dans le système qui les réunit ne se lisent pas de la même manière si les relations sont basées sur une interprétation des contextes dans le texte narratif, ou sur une distance tenant compte des positions dans le texte, c'est-à-dire uniquement de la forme du récit. De telles mesures de distance peuvent reposer sur les mots, les phrases, les paragraphes, ou, dans notre cas, sur les pages.

Pour construire le réseau, nous recensons les co-occurrences des entitéspersonnages à partir des occurrences recensées dans l'index. Nous sommes tributaires de l'édition choisie, ainsi que des conventions adoptées par l'indexeur ${ }^{9}$, par exemple d'inclure les auteurs cités.

\subsection{Affinement}

Nous ne suivons pas le scénario simpliste consistant à associer deux personnages s'ils apparaissent sur la même page ${ }^{10}$, car cette approche ne lierait pas deux personnages apparaissant par exemple dans la même phrase, l'un en bas

\footnotetext{
${ }^{6} \mathrm{Ou}$ centralité de pouvoir, ou centralité de Bonacich.

${ }^{7}$ Pour permettre la résolution d'un système de $\mathrm{n}$ équations à $\mathrm{n}$ inconnues, pour $\mathrm{n}$ le nombre de sommets du réseau.

${ }^{8}$ Sur ce point, consulter la littérature sur les algorithmes de détection de communautés, par exemple (Fortunato, 2010).

${ }^{9}$ Par exemple, l' « index des noms de personnes » des Confessions dans l'édition Classiques Garnier Poche (2011) contient des entrées pour noms tels que Minerve ou Orphée.

${ }^{10} \mathrm{Ce}$ qui correspond à l'information brute telle qu'elle est fournie par un index.
} 
d'une page et l'autre au sommet de la suivante. Pour générer le réseau des co-occurrences à partir de l'index des personnages, nous le transformons en un index de couples de pages : un personnage apparaissant sur une page $\mathrm{n}$ dans le premier index appartient dans le second index aux couples de pages $\{n-1, n\}$ et $\{\mathrm{n}, \mathrm{n}+1\}$. Cette méthode permet de substituer aux pages du premier index un système d'occurrences couplées et superposées ${ }^{11}$. Elle tient mieux compte de la proximité entre les personnages : deux sommets apparaissant sur la même page (co-occurrence forte) sont ainsi considérés par construction comme plus proches que deux sommets sur des pages consécutives (co-occurrence faible).

Cette approche par couples de pages résout le problème du format trop strict de la page, mais introduit du "bruit" : en admettant des occurrences plus larges, les co-occurrences qui en sont déduites sont également plus nombreuses. Pour pallier à ça, nous fixons un seuil à atteindre pour qu'une relation soit prise en compte. En effet, lors de la compilation des co-occurrences, nous définissons un attribut de poids pour chaque relation, égal à la somme des co-occurrences faibles (dont la valeur ajoute une unité) et fortes (dont la valeur ajoute deux unités ${ }^{12}$ ). Une valeur minimale du poids égale à trois est requise pour la création de l'arête correspondante. La relation entre deux personnages atteint ce seuil lorsqu'ils partagent au moins une co-occurrence forte et une co-occurrence forte ou faible, ou au moins trois co-occurrences faibles.

En résumé, un personnage est retenu dans le modèle s'il existe au moins un autre personnage dans le roman partageant avec lui une relation jugée significative. C'est l'imbrication d'un personnage dans l'espace d'un ou d'autres personnages qui importe lors de la génération du réseau et de la définition des personnages à retenir. Ainsi, l'apparition dans des événements impliquant d'autres personnages, relatés sur plusieurs pages, garantit la présence du personnage dans le réseau final.

Le réseau des co-occurrences obtenu ne conserve pas tous les personnages présents dans l'index. Une analyse de l'ensemble de tous les personnages de la narration est aussi possible : nous ne fixons alors pas de seuil. Ce réseau doit alors être interprété différemment, et les conclusions que nous en tirerions concerneraient d'autres questions, mais le problème du "bruit" demeurerait.

\subsection{Premières propriétés du réseau}

Le réseau que nous obtenons contient 227 personnages sur les 584 apparaissant dans le texte, ainsi que 617 arêtes. Il est simple et non-dirigé. Sur les 357 personnages non-retenus, 310 n'apparaissent qu'une seule fois dans le texte, 36 deux fois et 11 trois fois. En comparaison, aucun personnage n'apparaissant qu'une seule fois dans le texte n'est dans le modèle, mais 64 personnages apparaissant deux fois et 49 personnages apparaissant trois fois le sont. Dès lors, il faut prendre en compte le fait que les personnages situés en périphérie du réseau ne sont par nécessairement des personnages anecdotiques dans le texte, puisqu'ils font partie des moins de $40 \%$ retenus.

À ce stade, le réseau n'est pas connexe, car cinq couples de personnages gravitent hors de la composante géante ${ }^{13}$. Ces couples illustrent des événe-

\footnotetext{
${ }^{11}$ En effet, superposées car il n'y a plus de « vide » entre deux pages successives. Il n'est plus possible que deux personnages cités côte-à-côte ne puissent pas être considérés ensemble. ${ }^{12}$ Puisqu'une co-occurrence forte et la réunion de deux co-occurrences faibles.

${ }^{13}$ Le plus grand sous-ensemble connecté de sommets, c'est-à-dire qu'il existe un chemin
} 
ments narratifs importants, mais n'ayant pas ou trop peu d'interventions de personnages en commun avec les autres événements composant l'oeuvre. Pour des raisons de calcul, nous restreignons le réseau des co-occurrences de personnages à la composante géante, et mettons donc de côté dix sommets et cinq arêtes qui étaient déconnectés du reste du réseau. Le résultat obtenu est montré à la figure 1 .

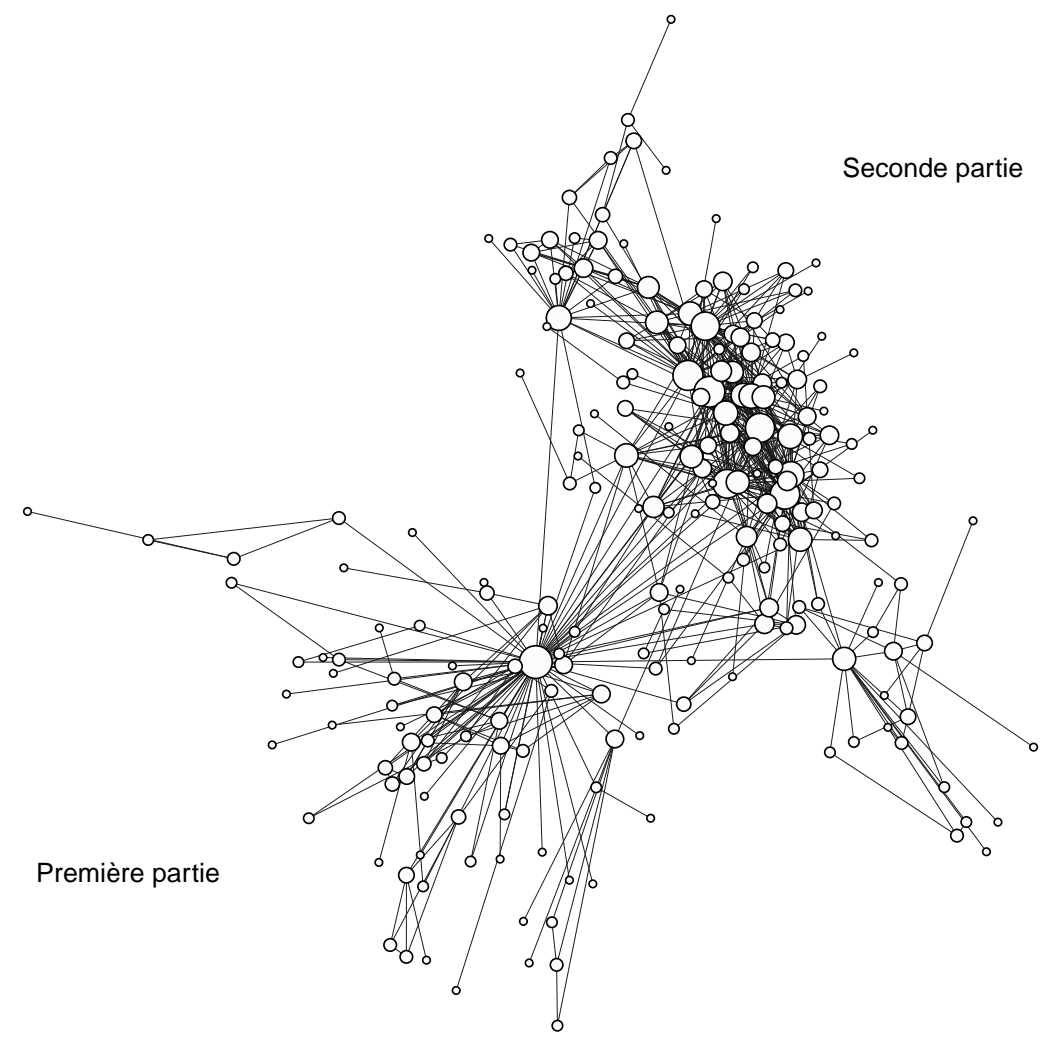

FiguRE 1 - Le réseau des co-occurrences des personnages, après restriction à la composante géante. Nous indiquons dans l'image où se situent les personnages des deux parties du livre. 16.5\% des personnages représentés dans ce réseau apparaissent dans les deux parties des Confessions.

\section{Structure narrative et centralités}

Nous avons exposé précédemment les divers indices de centralité nécessaires à cette étude. Ils nous permettent maintenant de décrire l'organisation de la structure narrative des personnages par l'auteur. Nous discutons les valeurs obtenues et en déduisons quelques résultats en se basant sur une interprétation de ces valeurs et de diverses propriétés du réseau. Attention toutefois à faire la distinction : certains des résultats présents dans cette section sont entièrement déduits du réseau et renseignent sur le texte, d'autres sont au contraire des illustrations par le réseau de faits tirés du texte. Ainsi, le rôle du réseau est

reliant chaque couple d'éléments de cette composante. 
de soulever des questions comme d'y répondre, il peut être variable expliquée comme explicative.

Des 217 sommets retenus dans le réseau des co-occurrences, le tableau 1 montre les douze sommets les plus centraux pour chacune des quatres mesures de centralité, réunis en un seul tableau afin de pouvoir les comparer. Nombre d'entre eux sont plusieurs fois parmi les plus centraux, d'où le nombre réduit de sommets présentés. Les sommets sont ordonnés selon leurs degrés.

\begin{tabular}{llrll}
\hline & Degré & Interm. & Harm. & V.propres \\
\hline Mme de Warens & $64(1)$ & $14926.69(1)$ & $34.90(1)$ & $0.190(18)$ \\
Thérèse Levasseur & $48(2)$ & $1231.19(11)$ & $26.29(9)$ & $0.793(4)$ \\
Mme de Luxembourg & $43(3)$ & $2969.67(3)$ & $29.66(2)$ & $0.475(7)$ \\
Denis Diderot & $40(4)$ & $2171.27(5)$ & $27.88(4)$ & $0.964(3)$ \\
Grimm & $38(5)$ & $1693.00(6)$ & $26.94(6)$ & $0.978(2)$ \\
Louise d'Épinay & $36(6)$ & $1282.83(8)$ & $25.64(11)$ & $1.000(1)$ \\
Duc de Luxembourg & $34(7)$ & $406.12(33)$ & $22.74(44)$ & $0.469(8)$ \\
Mme Dupin & $25(8)$ & $1295.08(7)$ & $27.16(5)$ & $0.277(11)$ \\
George Keith & $21(9)$ & $2198.60(4)$ & $26.33(7)$ & $0.081(31)$ \\
Comtesse d'Houdetot & $20(10.5)$ & $1079.13(12)$ & $26.32(8)$ & $0.637(5)$ \\
Jean Le Rond d'Alembert & $20(10.5)$ & $359.60(35)$ & $23.46(30)$ & $0.210(14)$ \\
M. et Mme Levasseur & $19(12)$ & $774.45(16)$ & $23.67(24)$ & $0.511(6)$ \\
Voltaire & $17(15)$ & $1259.88(10)$ & $27.91(3)$ & $0.117(24)$ \\
Comtesse de Boufflers & $17(15)$ & $125.62(68)$ & $22.77(43)$ & $0.203(15)$ \\
Comte de Montaigu & $16(17.5)$ & $3298.87(2)$ & $23.73(23)$ & $0.005(123)$ \\
Charles Pinot Duclos & $15(20)$ & $515.56(24)$ & $22.67(45)$ & $0.246(12)$ \\
Baron d'Holbach & $13(22.5)$ & $47.47(87)$ & $20.83(74)$ & $0.426(9)$ \\
Marquise de Verdelin & $13(22.5)$ & $474.45(26)$ & $25.00(12)$ & $0.052(46)$ \\
Saint-Lambert & $9(28)$ & $13.18(103)$ & $18.57(128)$ & $0.389(10)$ \\
M. de La Poplinière & $8(36)$ & $135.29(66)$ & $25.83(10)$ & $0.053(44)$ \\
Mme de Larnage & $7(48.5)$ & $1272.00(9)$ & $16.97(161)$ & $0.007(106)$ \\
\hline
\end{tabular}

TABLE 1 - Mesures de centralité des douze sommets les plus centraux pour chaque indice, réunis en un seul tableau et classés en fonction de leurs degrés. Entre parenthèses, leurs rangs relativement à chaque indice.

À l'aide du tableau 1 et de la figure 2, nous observons que la distribution des degrés décroit rapidement. En effet, quelques sommets possèdent un grand nombre de connexions, tandis que la majorité en possède peu : la médiane est égale à trois, et le minimum est égal à un. Il existe des sommets beaucoup plus importants dans la trame que d'autres.

La centralité d'intermédiarité et la centralité par vecteurs propres décroissent également rapidement. Dans ces deux cas, quelques sommets maximisent les propriétés mesurées respectivement par ces indices ${ }^{14}$, tandis qu'à l'opposé se trouvent des sommets pour lesquels les mesures sont nulles.

La centralité harmonique suit une distribution dont la densité est symétrique. Les valeurs les plus hautes sont attribuées aux sommets proches du centre du réseau, les valeurs les plus basses aux sommets en périphérie. La majorité se situe dans une zone à mi-distance. Pour cet indice, médiane et moyenne sont égales.

À partir de ces mesures, nous discutons les rôles narratifs de quelques per-

\footnotetext{
${ }^{14}$ La valeur maximale de la centralité par vecteurs propres est égale à un. Elle est toujours atteinte par au moins un sommet.
} 

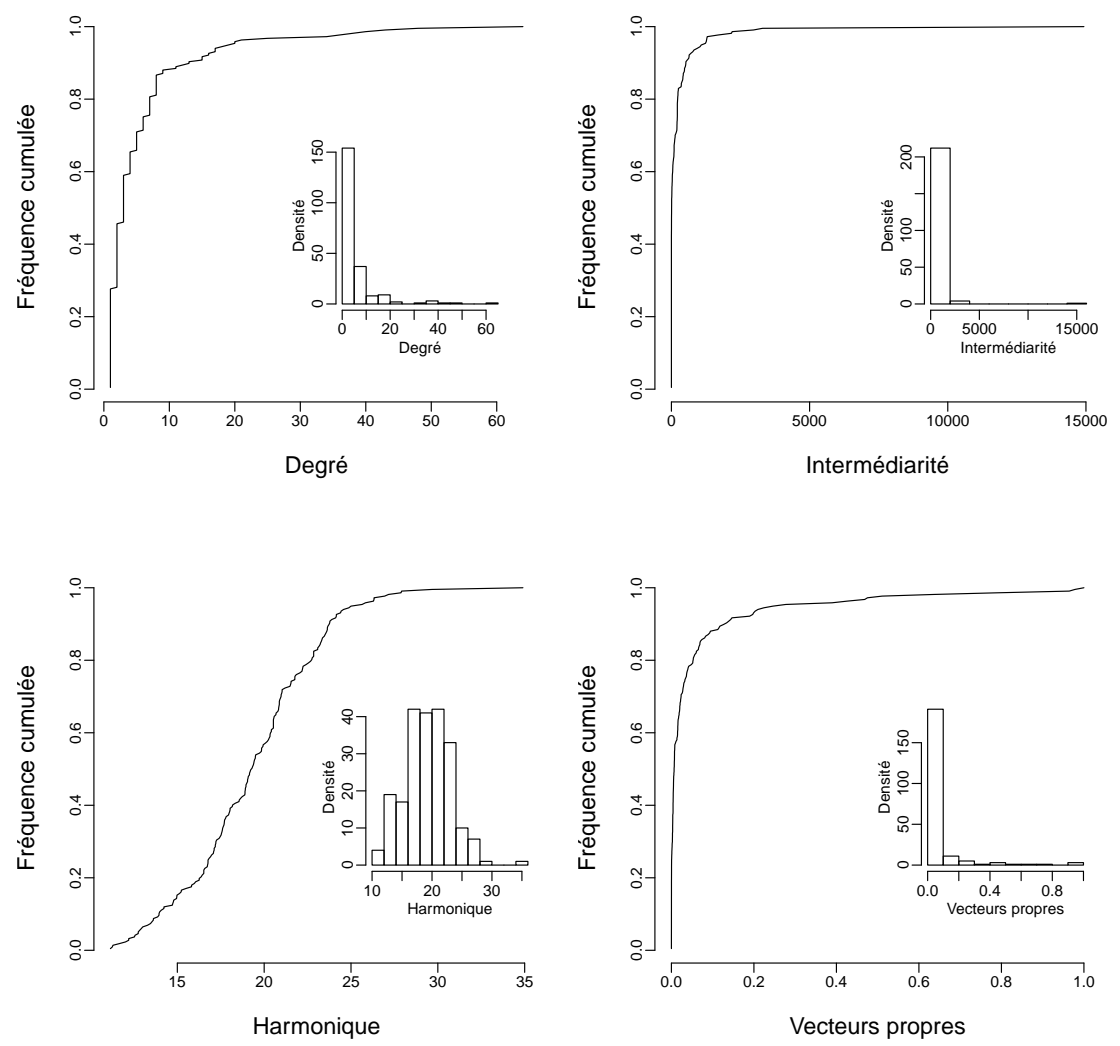

Figure 2 - Fréquences cumulées et densités des mesures de centralité.

sonnages ou groupes de personnages dans le récit.

\subsection{Mme de Warens}

Les Confessions sont divisées en deux parties, chacunes divisées en six chapitres. Il s'avère que Mme de Warens obtient des scores significativement plus élevés que ceux des autres personnages pour les centralités de degré, harmonique et d'intermédiarité. Elle est la figure principale de la première partie des Confessions, apparaissant majoritairement dans les chapitres deux à six ${ }^{15}$. Les sommets suivants dans les classements selon les indices correspondent tous à des personnages apparaissant pour la plupart dans la seconde partie, plus dense, comportant plus de personnages et induisant plus de relations dans le réseau des co-occurrences.

Il y a une absence de concurrence dans la première partie, construite autour du personnage de Mme de Warens. Son rôle de mentor auprès de Rousseau est décrit dans le texte. Il apparaît lié à de nombreux autres personnages dans le réseau, et par là à de nombreuses communautés. Son rôle de passeur narratif, qui se traduit par une centralité d'intermédiarité très forte, est visible dans la figure 3 .

\footnotetext{
${ }^{15} 87 \%$ de ses apparitions se font dans la première partie. Dans la seconde partie, elle apparaît au moins une fois dans tous les chapitres, sauf au chapitre dix où elle n'apparaît pas.
} 


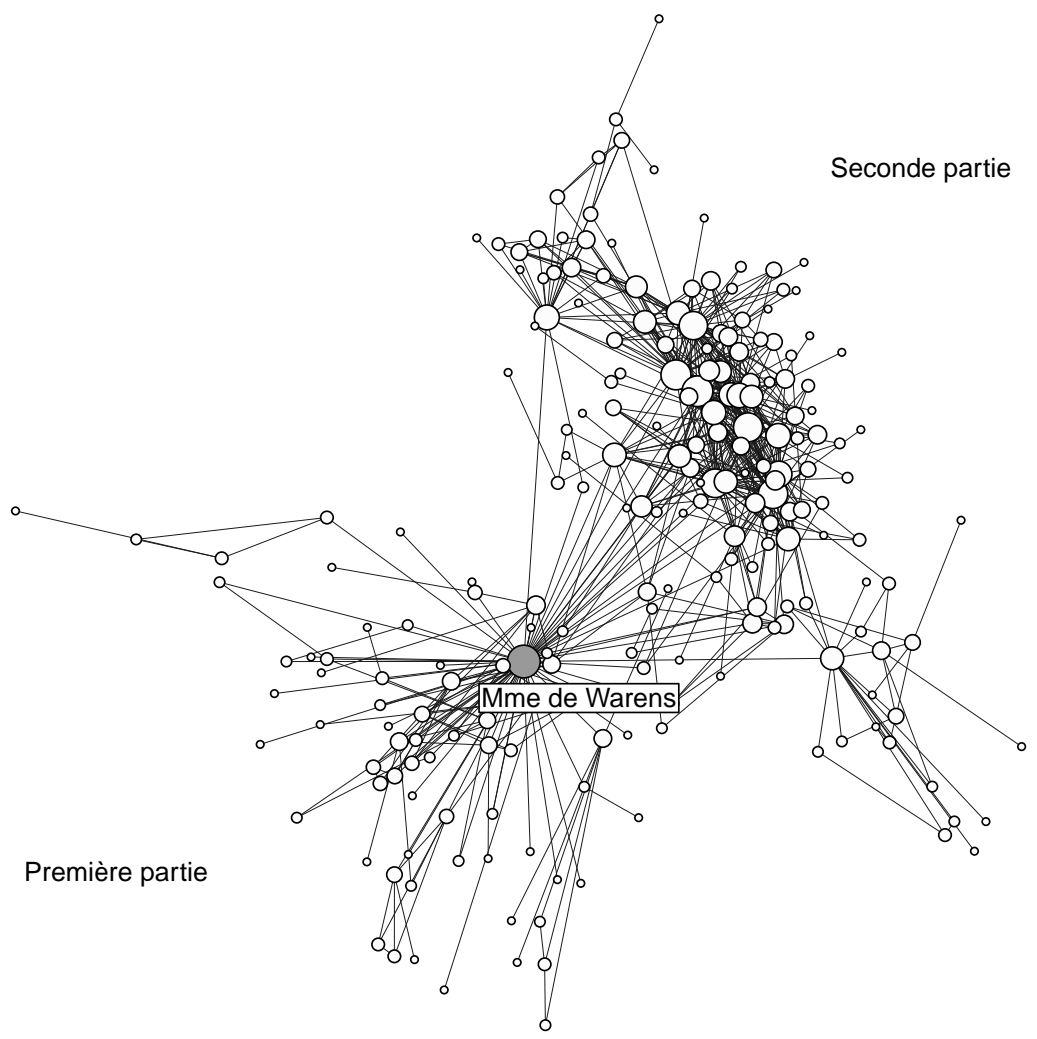

FigURE 3 - Le réseau des co-occurrences des personnages, avec mise en évidence du sommet représentant Mme de Warens.

Un indice fait exception dans ces classements : il s'agit de la centralité par vecteurs propres. En effet, cet indice renforce les scores des sommets dont les voisins ont des scores élevés. Le personnage de Mme de Warens est peu présent dans la seconde partie du roman, là où la vie sociale de Rousseau et des personnages qu'il côtoie est plus dense. Cette absence de relations vers des personnages issus de communautés fortes se trahit dans son score de centralité par vecteurs propres relativement bas. Il s'agit d'une différence importante dans les approches narratives respectives des deux parties : dans la première, Mme de Warens présente Rousseau à des personnages auxquels il ne s'attache jamais longtemps dans la narration. Les événements qui les contiennent sont souvent disjoints. Cela se retrouve dans le réseau, où ils occupent des positions relativement secondaires, traduites par des scores de centralité bas. Pour autant, cela ne signifie pas qu'ils sont moins présents en termes d'occurrences.

\subsection{Le méta-personnage du complot}

Des soupçons de vol d'éléments de sa correspondance, ainsi que la publication de textes et pamphlets ternissant son image convainquent Rousseau de l'existence d'un complot le visant. Alors qu'il a terminé l'écriture de la première partie des Confessions, il entre à cette époque dans une période marquée par la paranoïa (Voisine, 2011, p. CLIII). Une manifestation de ce trouble est la dénonciation 
qu'il fait des auteurs dudit complot dans le texte, en particulier dans sa manière d'y structurer les personnages.

Nous avons vu que la centralité par vecteurs propres ne considère pas Mme de Warens comme centrale (voir tableau 1). Au contraire, et à l'exception de ses intimes ${ }^{16}$, les personnages obtenant des scores élevés sont principalement des membres de ce complot. Cet indice met en évidence un sous-groupe plus dense, et possédant des propriétés communes, du réseau des personnages. Sur la base de leurs scores, nous détectons notamment comme membres de celui-ci Louise d'Épinay, Friedrich Melchior Grimm, Denis Diderot, Jean le Rond d'Alembert, le baron d'Holbach, et la comtesse de Boufflers.

$\mathrm{Au}$ niveau de la narration, ces personnages forment un méta-personnage composé de figures interchangeables. Cette image se justifie par le fait que l'existence de ce groupe et de ses ramifications demeure si nous en retirons un des personnages, leurs voisinages étant en partie communs. Dans le texte, Rousseau décrit ces individus à plusieurs reprises de manière groupée, tel un ennemi sans visage. Ce fait se trouve vérifié lorsque nous étudions la contraction des sommets représentant les membres du complot en un seul sommet. Cette construction consiste à remplacer les sommets choisis par un seul sommet héritant de toutes les connexions de ceux qui le composent. Ce que nous obtenons est la représentation du méta-personnage du complot dans le réseau. Elle nous permet d'observer l'emprise que possède cette réunion d'espaces de personnages au sein du système entier. Les mesures de centralité de ce sommet sont proches de celles du sommet représentant Mme de Warens (à l'exception toujours de la centralité par vecteurs propres, voir tableau 2).

\begin{tabular}{llrll}
\hline & Degré & Interm. & Harm. & V.propres \\
\hline Complot & $83(1)$ & $7025.94(2)$ & $31.17(2)$ & $1.000(1)$ \\
Mme de Warens & $61(2)$ & $12842.92(1)$ & $33.29(1)$ & $0.205(11)$ \\
\hline
\end{tabular}

TABLE 2 - Mesures de centralité du sommet représentant Mme de Warens et du sommet réunissant les personnages du complot. Entre parenthèses, leurs rangs pour chaque indice respectivement.

Leurs positions en premières ou secondes places nous permet d'affirmer que l'importance narrative donnée au groupe de personnages derrière le complot est similaire dans la seconde partie à celle donnée à Mme de Warens dans la première partie. Néanmoins, il ne s'agit pas de prétendre que leurs rôles narratifs sont équivalents. Nous montrons dans la figure 4 la répartition des sommets des personnages décrits comme adhérant à ce complot. Nous observons la séparation du réseau des co-occurrences en deux parties, l'une disposée autour de Mme de Warens (voir figure 3), l'autre, plus diffuse, répartie le long des personnages du complot $^{17}$.

\subsection{Typologie des espaces de personnages}

Dans cette section, nous analysons les mesures de centralité de quelques personnages plus en détail. Nous montrons ainsi comment l'analyse des réseaux littéraires permet de déduire des assertions sur les aspects narratifs d'un texte. Les mesures de centralité mentionnées dans la suite de cette section se consultent

${ }^{16}$ Les Levasseur, M. et Mme de Luxembourg, la comtesse d'Houdetot, ...

${ }^{17}$ L'algorithme de répartition des sommets est donné par (Fruchterman et Reingold, 1991). 


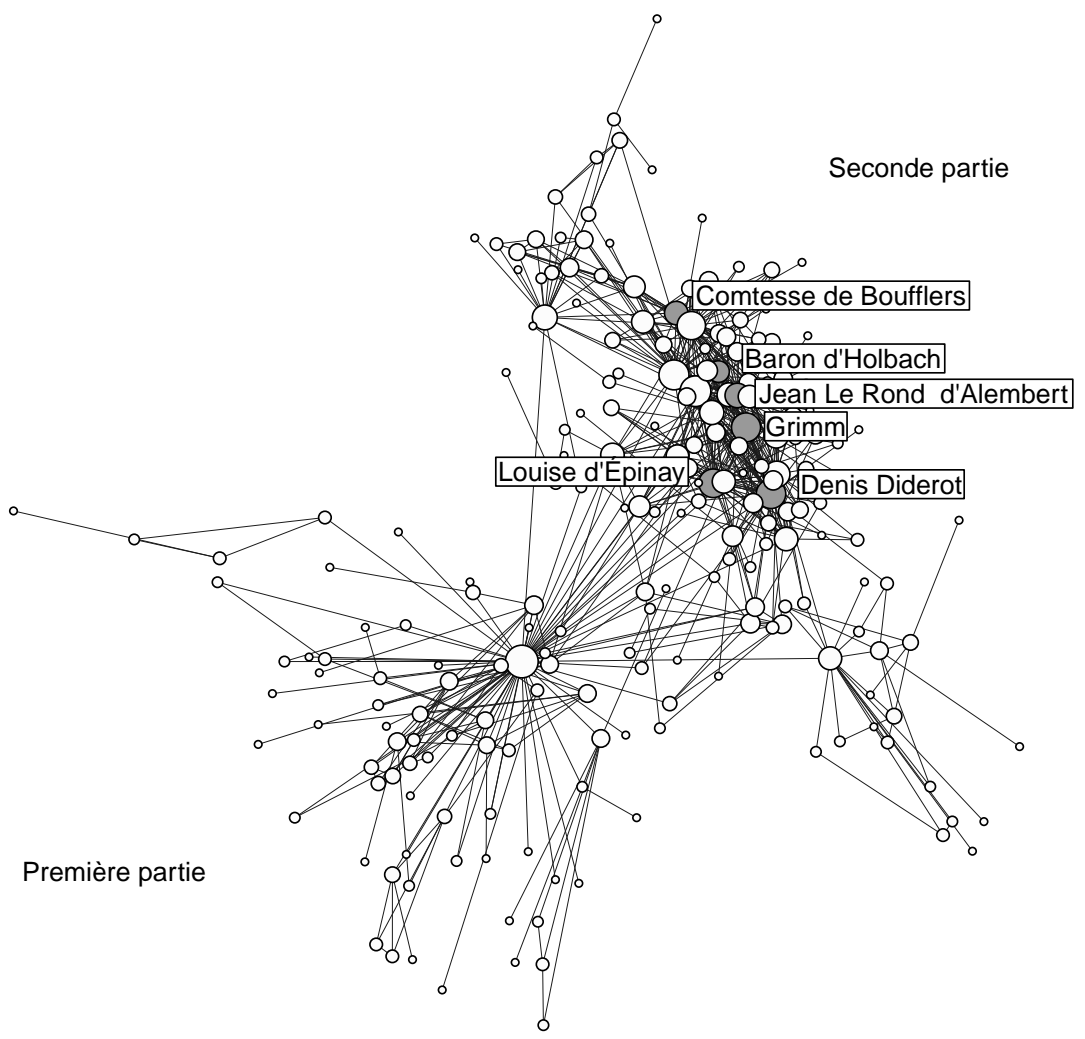

FigURE 4 - Le réseau des co-occurrences des personnages, avec mise en évidence de membres supposés du complot.

au tableau 1.

\subsubsection{Le baron d'Holbach, en retrait}

Le baron d'Holbach est un des personnages que Rouseau soupçonne d'appartenir au complot. Il apparait sur dix-huit pages, toutes dans la seconde partie des Confessions. Sa centralité de degré est élevée en comparaison des autres sommets du réseau, ce qui signifie qu'il apparaît aux côtés de nombreux autres personnages et qu'il est impliqué dans cette société des personnages du roman. Cependant, pour ce qui est des centralités harmonique et d'intermédiarité, il se trouve en retrait. Ces indices traduisent pourtant des formes d'influence au sein du réseau. Ainsi, par ses choix narratifs, Rousseau présente ce personnage comme un suiveur : présent et bien connecté, il est peu influent. S'il fallait déduire des Confessions quel personnage Rousseau place à la tête du complot, il ne s'agirait probablement pas du baron d'Holbach.

\subsubsection{Louise d'Épinay - Friedrich Melchior Grimm - Denis Diderot}

Ces trois personnages ont été parmi les plus proches amis de Rousseau, avant que ses relations avec chacun d'entre eux ne se détériorent. Ils possèdent, dans 
cet ordre, les trois premiers scores de centralité par vecteurs propres, et sont également considérés comme centraux selon tous les autres indices. Ainsi, nous en déduisons qu'ils occupent des positions narrativement influentes. Leur portée s'étend au-delà du groupe des "comploteurs" auquel ils appartiennent. Ils sont connectés à de nombreux personnages présents tout au long de la seconde partie des Confessions, ce qui dénote une influence potentiellement importante sur la vie de Rousseau, si l'on admet que celui-ci n'a pas consciemment mis en scène ce phénomène lors de la rédaction des Confessions.

\subsubsection{Le comte de Montaigu et George Keith, localement centraux}

Le comte de Montaigu occupe un type de position quasiment unique dans le réseau, ce que l'on remarque facilement dans la figure 5. Sa centralité d'intermédiarité est élevée au point de le placer juste derrière Mme de Warens tandis que les autres indices ne le mettent pas en évidence. Cela s'explique par sa position d'ambassadeur de France à Venise, alors que Rousseau travaille pour lui comme secrétaire. À une exception près, il n'est mentionné que dans le chapitre sept, qui comprend justement les événements prenant place à Venise. Dans le réseau, il joue donc un rôle de relais par ses liens en France entre les personnages vénitiens et les personnages des autres événements relatés dans Les Confessions. Il est à l'écart des personnages constituant le noyau du réseau, donc doit être caractérisé comme personnage secondaire à l'échelle de l'ensemble du récit. Cependant, il émerge en leader d'un groupe clairement défini de sommets, et selon ce point de vue occupe une position centrale, reléguant à des rôles secondaires les autres personnages au sein de ce contexte.

De manière moins marquée, George Keith (alias Milord Marechal) occupe au chapitre douze ${ }^{18}$ une position narrative similaire à celle du comte de Montaigu. Sa centralité d'intermédiarité est très élevée, sa centralité par vecteurs propres très basse, et dans la figure 5 il apparaît dans un groupe à l'écart. Cependant, des connexions nombreuses entre les personnages de cette communauté des événements en Suisse ${ }^{19}$ et les autres personnages de la seconde partie diminuent l'influence de George Keith sur ce groupe. Ces différences expliquent le contraste moins marqué entre les rangs de ses mesures de centralité qu'entre ceux du comte de Montaigu. Ce constat est peut-être même à relier avec la proximité géographique de la Suisse et des divers lieux visités en France, s'il s'avérait qu'elle augmente la densité du réseau des co-occurrences autour de George Keith, au contraire de Venise, plus distante.

\section{Conclusion}

Dans cet article, nous avons proposé un cadre théorique à l'analyse des réseaux littéraires et montré comment cette approche quantitative était une continuation de la théorie des personnages d'Alex Woloch. Puis, nous avons illustré notre propos à l'aide des Confessions de Jean-Jacques Rousseau.

L'utilisation d'un index des personnages est une manière parmi de nombreuses valables de proposer une analyse "sans ouvrir le livre". Nous avons

\footnotetext{
${ }^{18}$ Qui contient toutes ses occurrences (23), sauf une au chapitre deux qui n'est pas significative.

${ }^{19}$ Plus précisément Neuchâtel.
} 


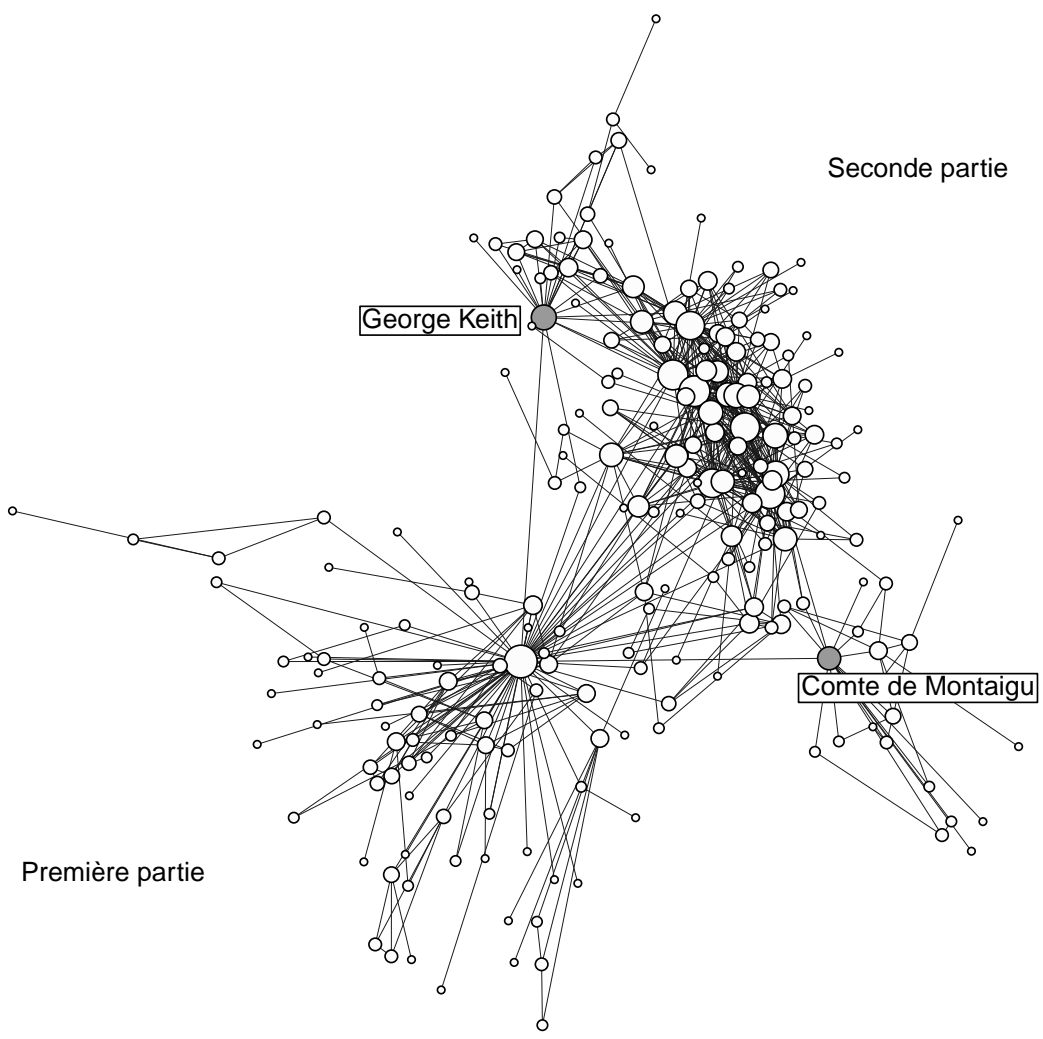

FiguRE 5 - Le réseau des co-occurrences des personnages, avec mise en évidence du comte de Montaigu et de George Keith.

$\mathrm{pu}$ proposer une visualisation des interactions narratives entre personnages $\mathrm{du}$ texte, puis poser des questions simplement à partir du réseau. La visualisation du réseau a également permis d'illustrer des faits ou propriétés connus du texte. Un développement possible serait de partir de l'histoire et des faits avérés, pour étudier comment l'auteur les rend dans le texte final.

De l'analyse des réseaux sociaux, nous avons principalement utilisé le concept de centralité, à travers ses indices les plus répandus. Nous avons montré comment, au centre de chacune des parties du roman, Rousseau place un personnage autour duquel il structure le propos : la première fois un personnage unique, mentor à l'aura positive, la seconde fois un personnage multiple, fourbe et destructeur. Puis, finalement, nous avons esquissé une typologie de rôles narratifs que l'on peut déduire de cette analyse de réseaux, en nous veillant toutefois à ne pas surinterpréter l'objet mathématique et le pousser à dire ce que nous souhaitions entendre.

\section{Bibliographie}

Apoorv Agarwal, Augusto Corvalan, Jacob Jensen et Owen Rambow : Social network analysis of alice in wonderland. NAACL-HLT 2012, pages 88-96, 2012. 
R. Alberich, J. Miro-Julia et F. Rossello : Marvel Universe looks almost like a real social network. arXiv :cond-mat/0202174, février 2002.

Mieke BAL : Narratologie. Essais sur la signification narrative dans quatre romans modernes. HES Publishers, Utrecht, 1984.

Alex BAVELAS : Communication patterns in task-oriented groups. Journal of the Acoustical Society of America, 22:725-730, 1950.

Claude Berge : Théorie des graphes et ses applications. Dunot, Paris, 1958.

Paolo Boldi et Sebastiano VIGNA : Axioms for centrality. arXiv :1308.2140 [physics], août 2013.

Andrea Bolioli, Matteo Casu, Maurizio Lana et Renato Roda : Exploring the Betrothed Lovers. In Mark A. Finlayson, Bernhard Fisseni, Benedikt Löwe et Jan Christoph MeIster, éditeurs : 2013 Workshop on Computational Models of Narrative, volume 32 de OpenAccess Series in Informatics (OASIcs), page 30-35, Dagstuhl, Germany, 2013. Schloss Dagstuhl-LeibnizZentrum fuer Informatik.

Phillip BONACICH : Factoring and weighting approaches to status scores and clique identification. The Journal of Mathematical Sociology, 2(1):113-120, 1972 .

Phillip Bonacich : Power and centrality : A family of measures. American Journal of Sociology, 92:1170-1182, 1987.

Stephen P. Borgatti : Centrality and network flow. Social Networks, 27(1):55 $-71,2005$.

Stephen P. Borgatti et Martin G. Everett : A graph-theoretic perspective on centrality. Social Networks, 28(4):466 - 484, 2006.

Stephen P Borgatti, Ajay Mehra, Daniel J Brass et Giuseppe LabiancA : Network Analysis in the Social Sciences. Science, 323(5916):892-895, février 2009 .

Ulrik Brandes, Sven Kosub et Bobo NiCK : Was messen Zentralitätsindizes? In Die Integration von Theorie und Methode in der Netzwerkforschung, pages 33-52. M. Hennig und C. Stegbauer (Hrsg.), Springer, 2012.

P. Mac Carron et R. Kenna : Network analysis of the Íslendinga sögur - the Sagas of Icelanders. The European Physical Journal B, 86(10):1-9, octobre 2013.

Pádraig Mac CARron et Ralph Kenna : Universal properties of mythological networks. EPL (Europhysics Letters), 99(2):28002, juillet 2012.

Steven R. Corman, B. Hunter Ball, Kimberly M. Talboom et Gene A. BreWER : Assessing Two-Mode Semantic Network Story Representations Using a False Memory Paradigm. In Mark A. Finlayson, Bernhard Fisseni, Benedikt LöwE et Jan Christoph MeIster, éditeurs : 2013 Workshop on Computational Models of Narrative, volume 32 de OpenAccess Series in Informatics (OASIcs), page 53-61, Dagstuhl, Germany, 2013. Schloss Dagstuhl-LeibnizZentrum fuer Informatik.

Alain Degenne et Michel Forsé : Les réseaux sociaux. Armand Colin, Paris, 1994. 
David K. Elson : Modeling narrative discourse. Thèse de doctorat, Columbia University, New York City, 2012.

Santo Fortunato : Community detection in graphs. Physics Reports, 486 (3-5):75-174, février 2010 .

Linton C. Freeman : Centrality in social networks : conceptual clarification. Social Networks, 1(3):215 - 239, 1978.

Thomas MJ Fruchterman et Edward M ReINGOLD : Graph drawing by forcedirected placement. Software : Practice and experience, 21(11):1129-1164, 1991.

Sebastian Gil, Laney Kuenzel et Caroline Suen : Extraction and Analysis of Character Interaction Networks From Plays and Movies. CS 224W final project report, Stanford, 2011.

Pablo M. GLeiser : How to become a superhero. Journal of Statistical Mechanics : Theory and Experiment, 2007(09):P09020, septembre 2007.

Martin Grandjean : Comparing the relational structure of the Gospels. Network Analysis as a tool for biblical sciences, 2013.

Marina Hennig, Ulrik Brandes, Jürgen Pfeffeer et Ines Mergel : Studying Social Networks : A Guide to Empirical Research. Campus Verlag, septembre 2012.

Sterling Hutchinson, Vivek Datla et Max M. Louwerse : Social networks are encoded in language. In Proceedings of the 34th annual conference of the cognitive science society, page 491-496, 2012.

Matthew L. Jockers : Macroanalysis. Digital methods and literary history. University of Illinois Press, mars 2013.

Donald E. KNuth : The Stanford GraphBase : A Platform for Combinatorial Computing, 2013.

Dirk Koschützki, Katharina Anna Lehmann, Dagmar Tenfelde-Podehl et Oliver Zlotowski : Advanced Centrality Concepts. In Ulrik Brandes et Thomas ErLEBACH, éditeurs : Network Analysis, volume 3418, pages 83-111. Springer, Berlin, Heidelberg, 2005.

Philippe LeJeune : Le pacte autobiographique : Signes de vie, volume 1. Seuil, 1975.

Franco Moretti : Network theory, plot analysis. New Left Review, 68, avril 2011.

Eric T. NAlisnick et Henry S. BAIRD : Character-to-Character Sentiment Analysis in Shakespeare's Plays. In Proceedings of the 51st Annual Meeting of the Association for Computational Linguistics, Sofia, Bulagria, août 2013a.

Eric T. NALisnick et Henry S. BAIRD : Extracting Sentiment Networks from Shakespeare's Plays. In 2013 12th International Conference on Document Analysis and Recognition (ICDAR), pages 758-762, 2013b.

M. E. J Newman et M. Girvan : Finding and evaluating community structure in networks. Phys. Rev. E, 69(2), 2004. 
Brian T. Pentland et Martha S. Feldman : Narrative Networks : Patterns of Technology and Organization. Organization Science, 18(5):781-795, octobre 2007.

Yannick RocHAT : Closeness centrality extended to unconnected graphs : the harmonic centrality index. Rapport technique, ASNA conference, Zürich, août 2009.

Britta Ruhnau : Eigenvector-centrality - a node-centrality? Social Networks, 22(4):357-365, octobre 2000.

Jeff RYDBERG-COX : Social networks and the language of greek tragedy. In Journal of the Chicago Colloquium on Digital Humanities and Computer Science, volume 1, 2011.

Gert SABIDussi : The centrality index of a graph. Psychometrika, 31(4):581603, décembre 1966.

Graham SACK : Character Networks for Narrative Generation. In Eighth Artificial Intelligence and Interactive Digital Entertainment Conference, 2012.

Graham Alexander SACK : Character Networks for Narrative Generation : Structural Balance Theory and the Emergence of Proto-Narratives. In 2013 Workshop on Computational Models of Narrative, volume 32, page 183-197, 2013.

Amelia Carolina Sparavigna : On Social Networks in Plays and Novels. The international journal of sciences, 2(10):20-25, 2013.

James Stiller, Daniel Nettle et Robin IM Dunbar : The small world of Shakespeare's plays. Human Nature, 14(4):397-408, 2003.

Jean-Jacques Voisine : Introduction. In Les Confessions. Garnier, Paris, 2011.

Stanley Wasserman et Katherine FAust : Social Network Analysis : Methods and Applications. Cambridge University Press, novembre 1994.

Alex Woloch : The One Vs. the Many : Minor Characters and the Space of the Protagonist in the Novel. Princeton University Press, 2003. 